\title{
COVID-19 vaccines: the dilemma when thinking as a patient
}

Sir,

What is the truth about the conspiracy theory of the COVID-19 microchip vaccine? The outbreak of the COVID-19 witnessed a vicious race of pharmaceutical firms to develop a vaccine that ends this disaster. Claims were forwarded that some firms funded by well-known foundations are in the process of developing such a vaccine so people wide world can be tracked. Such claims may have been based on Mr. Bill Gate's announcement in an interview that through vaccination "we will have some digital certificates" which would be used to show who had recovered, been tested and ultimately who received a vaccine but he made no mention of microchips. The conspiracy theory is spreading around the world and its supporters are on the increase among the public as well as many healthcare professionals. The spread of the virus has been attributed to the introduction of 5G technology and many religious leaders attributed the crisis to punishment from God to the spread of local wars and mass killing, torturing and killing of Muslims in China and Myanmar gay and lesbian movement and marriages.

On the other side the syringes for the COVID-19 injection were also accused of containing a microchip to track people. This was further strengthened by the United States Department of Defense awarding a $\$ 138$ million contract to ApiJect systems America in order to produce syringes for a COVID-19 vaccine once it become available. ${ }^{1}$ This was announced in collaboration with the department of health and human services, for "project jumpstart" and "RAPID USA," which together will dramatically expand U.S. production capability for domestically manufactured, medical-grade injection devices starting by October 2020 and to achieve the production of 500 million prefilled syringes (doses) by 2021. ApiJect claimed on its website's home page that the chip will be under the label of the syringe, on the outside and healthcare workers will have the option to tag it with their phone and that will allow them to upload a date, time and GPS location of each vaccination in real-time. In other words, it tracks when and where a vaccination was conducted. Such syringes are also claimed to be more economical and safer as it helps in avoiding reuse of syringes and cross contamination of vials which are already scarce and billions of them are needed as containers. The question that is inevitable is "What if a vaccine is developed for oral or nasal administration?

The race is so hot in search for a vaccine and so the antivirus movement and it is escalating. The latter is supported by the claims of many healthcare professionals and specialists that in their race to develop a vaccine, pharmaceutical firms are surpassing animal testing and some of the clinical phases required in the process.

The crisis of COVID-19 silently creeping affecting millions and causing the death of large numbers but the bells of accusation and contradictory claims in a nonorchestrated chaos causing tinnitus and the fear everywhere.

Going back to the conspiracy theory, the question was raised "could an injectable microchip be used to provide tracking in the manner contemplated by this theory $?^{2}$ The author claimed that this is not possible as a microchip has to be very tiny and floats in blood without causing embolism and be powered for a lifetime. This however, may somewhat be challenged by the rapid advances in nanotechnology and the secretively unpublished advanced technology that is going on in many governmental research institutes. The same author suggested that "some people may have misrepresented Gates funded into the idea of passively tracking vaccine deliveries by using nanoimprinted quantum dots. An approach to encode medical history on a patient using the spatial distribution of biocompatible, near-infrared quantum dots (NIR QDs) in the dermis. QDs are invisible to the naked eye yet detectable when exposed to NIR light. ${ }^{3}$ That could later be read by smartphone scanner." These findings suggest that intradermal QDs can be used to reliably encode information and can be delivered with a vaccine, which may be particularly valuable in the developing world and open up new avenues for decentralized data storage and biosensing (same above authors). Here the fact that we are all not necessarily protected against tracking while using smartphones and the social media networks. We send our location to friends and delivery personnel, and we use GPS to map and locate friends and facilities to reach.

Why the support went for syringes? Most authorities complain of shortage of glass vials for the "dream" vaccine once developed. Many authorities started claiming that the availability of glass vials is the main challenge as up to almost 15 billion doses are globally needed. It seems that efforts in the field of development of a vaccine against COVID-19 are not harmonized. Developing the vaccine is a priority and certainly manufacturers of medical glass all over the world can produce enough vials if given assurance that their production would be bought from them. Does such glass crisis is stirred up to advocate the "suspiciously accused" prefilled syringes? 
Is the cold war started already? Russia announced the start of the production of Sputnik V vaccine for COVID-19 virus despite the warnings and resignation of top Russian healthcare professionals. Specialists in the field warned against the long-term safety of the vaccine which they strongly believe that it was not considered while rushing to announce the still "suspicious" vaccine. Russia based its vaccine on the remarkable similarity of Middle East respiratory syndrome (MERS) to COVID-19. They used inactivated adenoviruses as vectors, delivering genetic material from S-protein, which forms the spike of coronavirus into a human cell to induce an immune response. The Russian health authorities claim that all volunteers who participated in the test of the vaccine developed reasonable immunity after three weeks of the first dose and the immunity was further raised by the second booster dose without any sign of adverse effects on any of the volunteers. Among the challenges raised against Sputnik V is the fear that if the vaccine is only partially effective it may provoke mutation of COVID-19 virus. However, it must be noted here that mutation is rare among viruses as compared to bacterial pathogens.

Who will be the first to receive a newly developed COVID-19 vaccine? It is only logical to assume that the first to be inoculated are the frontline fighters of the virus including healthcare workers and people at high risk of contracting the viral infection. Strict cautionary measures must be taken when approving any newly developed vaccine since the disaster may dramatically be a tragedy as we will not only lose our arms but also our brave soldiers in the fight against COVID-19.
Suleiman I. Sharif ${ }^{1 *}$, Rubian S. Sharif ${ }^{2}$

${ }^{1}$ Department of Pharmacology, Faculty of Medicine, ${ }^{2}$ Department of Orthodontics, Faculty of Dentistry, University of Benghazi, Benghazi, Libya

*Correspondence to

Dr. Suleiman I. Sharif,

E-mail: sharifsi@sharjah.ac.ae

\section{REFERENCES}

1. US-Department of Defense (DOD). DOD Awards \$138 Million Contract, Enabling Prefilled Syringes for Future COVID-19 Vaccine; 2020 Available at: https://www.defense.gov/Newsroom/Releases/Releas e/Article/2184808/dod-awards-138-million-contractenabling-prefilled-syringes-for-future-covid19/source/GovDelivery/. Accessed on 20 November, 2020.

2. Hruska J. Why Do $44 \%$ of Republicans Believe Bill Gates Will Use Coronavirus Vaccines to Inject Them with Microchips? Extreme Tech, 2020. Available at: https://www.extremetech.com/extreme/310951republicans-bill-gates-coronavirus-vaccinesmicrochips. Accessed on 24 November, 2020.

3. McHugh KJ, Jing L, Severt SY, Cruz M, Sarmadi M, Jayawardena HSN et al. Biocompatible near-infrared quantum dots delivered to the skin by microneedle patches record vaccination. Sci Translational Med. 2019;11(523):eaay7162.

Cite this article as: Sharif SI, Sharif RS. COVID-19 vaccines: the dilemma when thinking as a patient. Int J Basic Clin Pharmacol 2021;10:609-10. 